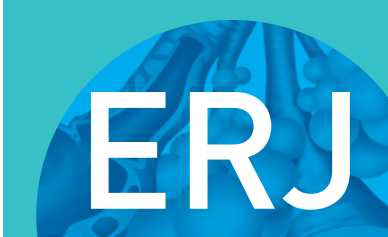

open research

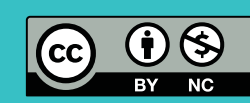

\title{
Predictors of loss to follow-up of tuberculosis cases under the DOTS programme in Namibia
}

\author{
Dan Kibuule (10), Philomein Aiases ${ }^{1}$, Nunurai Ruswa ${ }^{2}$, Timothy William Rennie ${ }^{1}$, \\ Roger K. Verbeeck ${ }^{1}$, Brian Godman ${ }^{3,4,5,6}$ and Mwangana Mubita ${ }^{1}$
}

Affiliations: ${ }^{1}$ School of Pharmacy, Faculty of Health Sciences, University of Namibia, Windhoek, Namibia. ${ }^{2}$ National Tuberculosis and Leprosy Programme, Ministry of Health and Social Services, Windhoek, Namibia. ${ }^{3}$ Division of Clinical Pharmacology, Karolinska Institutet, Stockholm, Sweden. ${ }^{4}$ Strathclyde Institute of Pharmacy and Biomedicial Sciences, University of Strathclyde, Glasgow, UK. ${ }^{5}$ Dept of Public Health Pharmacy and Management, School of Pharmacy, Sefako Makgatho Health Sciences University, Garankuwa, South Africa. ${ }^{6}$ Health Economics Centre, University of Liverpool Management School, Liverpool, UK.

Correspondence: Dan Kibuule, Dept of Pharmacy Practice and Policy, School of Pharmacy, University of Namibia, Private Bag 13301, Pioneers Park, Windhoek, Namibia. E-mail: dkibuuledunam.na

\section{ABSTRACT}

Background: In Namibia, one out of every 25 cases of tuberculosis (TB) is "lost to follow-up" (LTFU). This has impacted negatively on national efforts to end the disease by 2035 . The aim of this study was to determine the trends and predictors of LTFU under the directly observed treatment short-course (DOTS) programme in Namibia.

Methods: The study involved a retrospective longitudinal analysis of a nationwide cohort of TB cases registered under the DOTS programme in Namibia from 2006 to 2015. The trends and predictors of LTFU among cases in the National Electronic TB Register of the National TB and Leprosy Program were respectively determined by interrupted time series and multivariate logistic regression analyses using R-Studio software.

Results: Out of 104203 TB cases, 3775 (3.6\%) were LTFU. A quarter (26\%) of cases with poor outcomes were due to LTFU. The annual decline in cases of LTFU was significant between the first (2005-2010) and second (2010-2015) medium-term plan period for TB programme implementation $(\mathrm{p}=0.002)$. The independent predictors of LTFU were male sex $(\mathrm{p}=0.004)$, 15-24 years age group $(\mathrm{p}=0.03)$, provider of treatment $(p<0.001)$, intensive phase $(p=0.047)$ and living in border/transit regions $(p<0.001)$. HIV co-infection and TB regimen were not significant predictors of LTFU.

Conclusions: There were declining trends in LTFU in Namibia. DOTS programmes should integrate socioeconomic interventions for young and middle-aged adult male TB cases to reduce LTFU.

@ERSpublications

Loss to follow-up of tuberculosis patients is an important barrier to ending TB in high-TBburden countries. The integration of social welfare among young and/or middle-aged men is critical in reducing loss to follow-up of TB patients. http://bit.ly/395WfBM

Cite this article as: Kibuule $\mathrm{D}$, Aiases $\mathrm{P}$, Ruswa $\mathrm{N}$, et al. Predictors of loss to follow-up of tuberculosis cases under the DOTS programme in Namibia. ERJ Open Res 2020; 6: 00030-2019 [https://doi.org/10.1183/23120541.00030-2019]. 


\section{Introduction}

Tuberculosis (TB) has had a devastating impact on public health in Africa $[1,2]$. In 2018, out of the 10.0 million cases notified, $24 \%$ were from the sub-Saharan countries [3]. Of concern is that the region accounts for $80 \%$ of the 1.8 million estimated annual deaths related to TB; this is a disproportionate impact in terms of mortality [4]. Namibia, with a case notification rate of 442 cases per 100000 , is ranked fifth among countries with highest burden of TB [5, 6]. However, the universal access to community-based TB care through the Stop-TB and End-TB strategies implemented since 2005 has improved case identification and treatment outcomes in Namibia [7].

Nevertheless, the gradual rise in incidence of drug-resistant TB (DR-TB) and poor treatment outcomes such as "lost-to-follow-up" (LTFU) (i.e. an interruption of TB treatment for at least two consecutive months) and death [7-10] are major barriers to ending TB in Namibia [3, 11-14]. For instance, the incidence of LTFU among notified cases in Namibia increased from 4\% in 2014 to $10 \%$ in 2015. Studies in other low and middle-income countries (LMICs) such as India and Malaysia estimate higher incidences of LTFU at 19.2 and $24 \%$, respectively $[15,16]$. In addition, LTFU is an important risk factor for re-emergence of TB strains resistant to first-line anti-TB drugs [6]. In 2014, an estimated 300000 cases of multidrug-resistant (MDR) TB (i.e. resistance to backbone first-line anti-TB medicines, rifampicin and isoniazid) were notified globally [17]. In the same year Namibia notified 137 MDR-TB and 6 extensively drug-resistant (XDR) TB (i.e. MDR-TB with resistance to second-line TB drugs, aminoglycosides and/or fluoroquinolones) cases [10].

The significance of risk factors of LTFU (i.e. patient demographics, socioeconomic status, directly observed treatment, short-course (DOTS) programme, clinical covariates, TB treatment regimen and HIV co-infection) on LTFU have been contested across countries [10, 14, 15, 18-24]. In Namibia, despite universal coverage of high-quality DOTS, little is known about the impact of these factors on poor treatment outcomes and, in particular, LTFU, hence, the current study intended to determine the prevalence and determinants of LTFU in the Namibian context.

\section{Methods}

\section{Study design and population}

The target population was all the new and retreatment cases with drug-sensitive TB initiated on first-line anti-tuberculosis DOTS regimens. The accessible population was TB cases registered in the National Electronic Tuberculosis Register (ETR) database over a 10-year period from 2006 to 2015. The study included all 104300 TB cases registered in the ETR; 3775 of these were LTFU. A patient was considered LTFU if TB treatment was interrupted for 2 months or more [10, 17]. A retrospective cohort analysis for trends and predictors of LTFU was conducted. Quarterly trends in LTFU of TB cases in Namibia were analysed as a proportion of the total number of TB cases registered for each quarter. The main outcome measure was the effect size (i.e. odds ratio) for predictors for LTFU compared to TB cases that had a successful treatment outcome (i.e. cured or completed treatment). The study excluded all TB cases whose treatment outcome had not been registered in the ETR at the time of the study and patients with poor treatment outcome other than LTFU, (i.e. died, transferred out, treatment failure) (figure 1).

\section{Medium-term plans for TB in Namibia}

Since 2004, Namibia has implemented the DOTS strategy under a national strategic plan on TB implemented under 5-year medium-term plans (MTPs) to improve outcomes. The first MTP was implemented between 2004-2009, with the main goal of scaling up DOTS to all public health facilities in Namibia, with the main goal of achieving $85 \%$ treatment success rate from $65 \%$, and to strengthen detection and management of TB cases. The second MTP-II was developed to address gaps in MTP-I and was implemented between 2015 and 2017 to meet the World Health Organization (WHO) and national targets for TB. This focused mainly on the expansion and enhancement of access to quality TB treatment, bacteriological testing and community-based interventions for reduction of cases LTFU. Thus, the difference in the interventions served as discriminates to assess the effectiveness the two mid-term policy strategies for time-series analysis $[8,9]$

\section{Procedure and data analysis}

Data on treatment outcome, patient sociodemographic, clinical and treatment characteristics were extracted from the ETR and exported to R-Studio software (version 3.3.2) for quantitative analysis. The main outcome measures were an odds ratio of a TB case registered under DOTS programme getting LTFU relative to those with a successful treatment outcome (i.e. cured or completed). The covariates included in the model were patient characteristics, clinical/diagnostic, treatment and DOTS programmatic characteristics. The patient covariates were age, sex and region of residence. The clinical covariates were diagnostic or laboratory classification of the $\mathrm{TB}$ case, sputum conversion at 2 months and HIV 


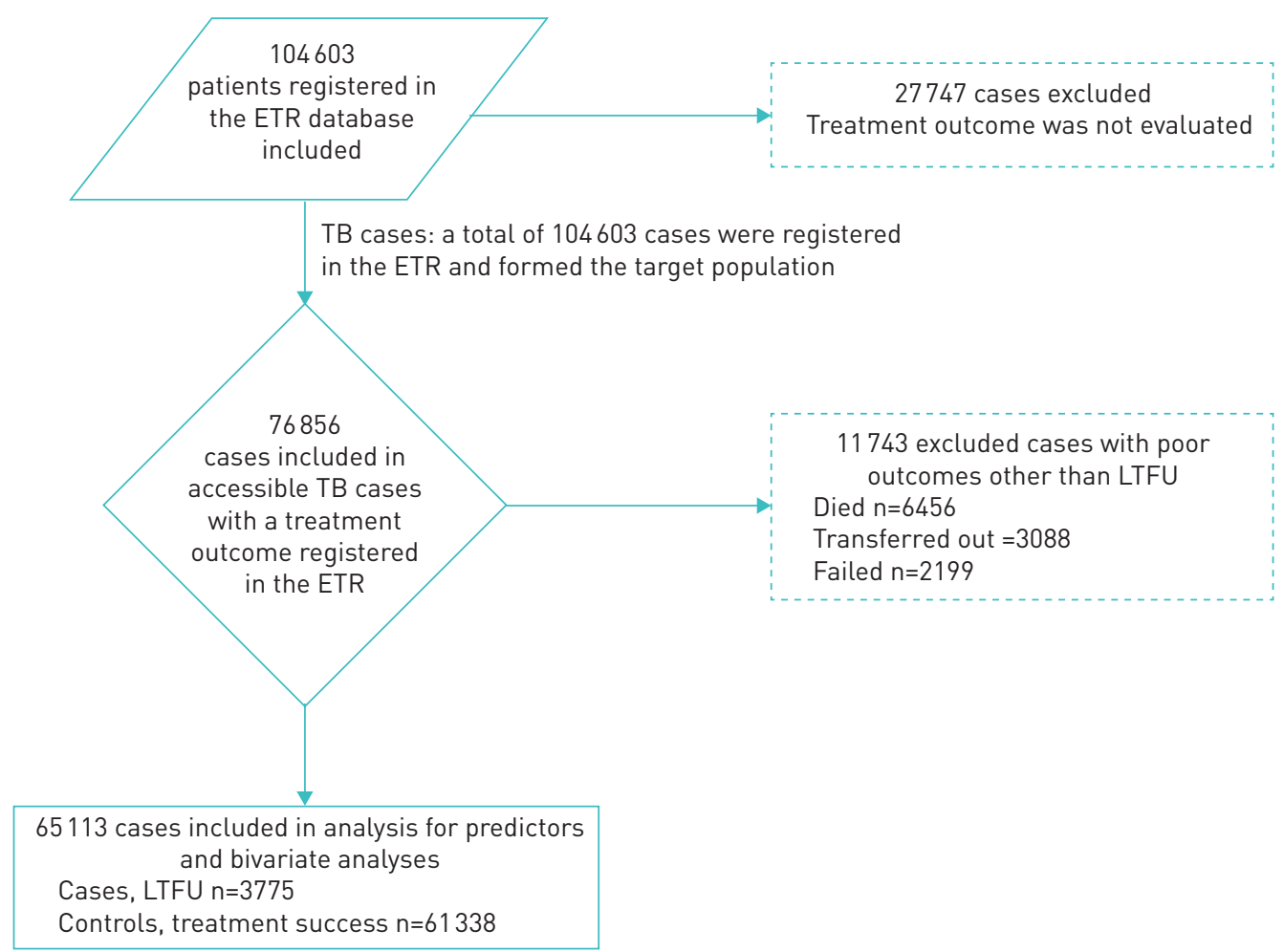

FIGURE 1 Flowchart for tuberculosis (TB) cases included in the analysis. ETR: electronic tuberculosis register; LTFU: lost to follow-up.

co-infection. The programmatic covariates were the TB strategies implemented (i.e. the first and second MTP for TB and leprosy), DOTS support category and level of health facility. Other clinical covariates pertained to the treatment regimens and therapy included the DOTS regimen initiated, anti-retroviral treatment (ART) regimen, type of DOTS provider, and previous prophylaxis with co-trimoxazole preventive therapy (CPT) and isoniazid preventive therapy (IPT). An interrupted time-series analysis was performed to determine the changes in level and trend of LTFU among TB cases registered in the DOTS programme during the implementation of MTP-I (2004-2009) and MTP-II (2010-2015) medium-term strategies for TB in Namibia. The following segmented regression model was used:

$$
Y_{t}=\beta_{0}+\beta_{1} T+\beta_{3} T X_{t}+e_{t}
$$

Where, $Y_{t}$ is the outcome (i.e. proportion of patients LTFU at time $t$ ), $T$ is the time (in years) elapsed since the start of the study, $X_{t}$ is a dummy variable indicating the pre-intervention period (coded 0 ) or the post-intervention period (coded 1); $\beta_{0}$ estimates the baseline outcome at $T=0 ; \beta_{1}$ is an estimate of the pre-intervention outcome trend (i.e. the change in outcome with time); $\beta_{2}$ is an estimate of the change in outcome immediately after the intervention (i.e. compared to the outcome at the end of the pre-intervention period); $\beta_{3}$ estimates the change in the post-intervention outcome trend compared to the pre-intervention outcome trend; $e_{t}$ represents the random variability not explained by the model. Adjustment for serial autocorrelation was carried out by using the Durbin-Watson statistic and by including an autocorrelation parameter in the segmented regression model if necessary.

Bivariate analysis using Chi-squared test or crude odds ratios was used to identify factors associated with LTFU. Significant factors were subsequently included in the multivariate logistic regression analysis to adjust odds ratios for confounding for independent predictors for LTFU and to elaborate on the relationships between multiple variables. The level of significance for the bivariate and multivariate analyses for a $95 \%$ confidence interval was set at a type I error $\alpha$ of 0.05 and $\beta$ of 0.20 (power $80 \%$ ) to detect a significant odds ratio [18].

\section{Ethics}

The research and ethics committees of the Ministry of Health and Social Services (MoHSS) (17/3/3/ November 2015) and University of Namibia (SOM/114/2016) approved the study. The need for written 
informed patient consent was waived as the study used retrospective records in the ETR database. Patient-specific identifiers, such as names, were anonymized or were not extracted or included from the dataset to ensure confidentiality.

\section{Results}

A significant decline in the quarterly trends in LTFU between 2006 and 2015 of 2.5 cases per quarter $\left(\mathrm{R}^{2}=0.45\right)$ was observed (figure 2 and table 2$)$. There was a statistically significant decline in the cases of LTFU per quarter $-0.23 \%(\mathrm{p}<0.001)$ during MTP-I. The cases of LTFU increased slightly at start of MTP-II. The quarterly trend in LTFU significantly increased by $0.16 \%(p=0.044)$ during MTP-II relative to MTP-I (figure 2 and table 1).

Out of the 104603 TB cases registered, 3775 (3.6\%) were LTFU; this ranged between $6.7 \%$ and $34.4 \%$ by regions in Namibia. The prevalence of cases LTFU among patients with unsuccessful treatment outcomes was $24.3 \%$ (3775 of 15 518; table 2). The majority of patients LTFU were: male (66\%), new TB cases (74.4\%), aged between 25 and 34 years $(31.7 \%)$, registered at primary healthcare (PHC) clinics $(58.6 \%)$, with a diagnosis of pulmonary TB (82.4\%), initiated on the standard 2HRZE/4HRE, i.e 2 months of isoniazid/rifampicin/pyrazinamide/ethambutol combination continued with 4 months of isoniazid/ rifampicin/pyrazinamide/ethambutol regimen (73.6\%), and registered during the implementation of the first MTP for TB (53.1\%) (figure 3). One-third of cases of LTFU had TB/HIV co-infection (33.5\%) and the majority of sputum smears of LTFU cases were either negative or not evaluated $(55.0 \%)$ at the start of treatment. A higher number of patients who were LTFU had a guardian (i.e. family member/relative,

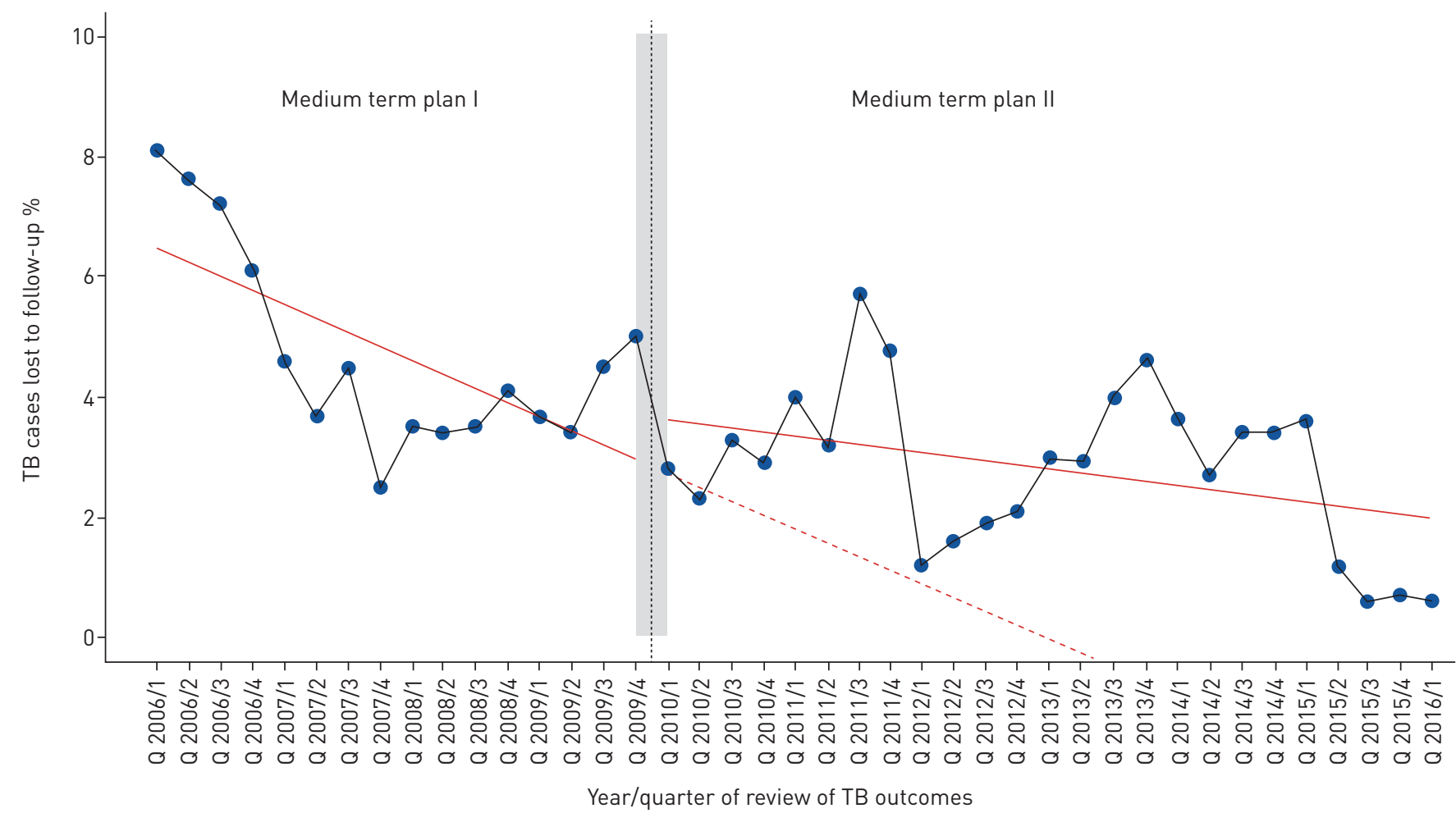

FIGURE 2 Trends of lost to follow-up cases in Namibia, 2006-2015. TB: tuberculosis; Q: quarter.

\begin{tabular}{|c|c|c|c|c|}
\hline Coefficients & Estimate $(95 \% \mathrm{CI})$ & SE & t-value & p-value \\
\hline Level of LTFU at start of MTP-I $\left(\beta_{0}\right)$ & $6.67(5.33-8.04)$ & 0.67 & 9.93 & $0.0001^{*}$ \\
\hline Trend in LTFU in MTP-I $\left(\beta_{1}\right)$ & $-0.23(-0.37--0.09)$ & 0.07 & -3.32 & $0.002^{*}$ \\
\hline Level change in LTFU in MTP-II $\left(\boldsymbol{\beta}_{2}\right)$ & $0.71(-0.93-2.35)$ & 0.81 & 0.88 & 0.385 \\
\hline Trend change in LTFU in MTP-II $\left(\boldsymbol{\beta}_{3}\right)$ & $0.16(0.004-0.32)$ & 0.08 & 2.08 & $0.044^{*}$ \\
\hline
\end{tabular}




\begin{tabular}{|c|c|c|}
\hline & LTFU & $\left(\chi^{2}=415, p=0.001\right)$ \\
\hline Omaheke & 25 & 372 \\
\hline Erongo & 264 & 1628 \\
\hline Zambezi & 62 & 269 \\
\hline Kavango West & 237 & 1023 \\
\hline Omusati & 295 & 1155 \\
\hline Ohangwena & 345 & 1272 \\
\hline Kavango East & 88 & 315 \\
\hline Oshana & 85 & 265 \\
\hline National & $3775(3.6 \%)$ & 11743 \\
\hline Hardap & 138 & 409 \\
\hline Karas & 263 & 766 \\
\hline Oshikoto & 446 & 1024 \\
\hline Kunene & 172 & 387 \\
\hline Khomas & 1032 & 2241 \\
\hline Otjozondjupa & 323 (34.4\%) & 617 \\
\hline
\end{tabular}

FIGURE 3 Prevalence of loss to follow-up (LTFU) among tuberuculosis cases by geographic region in Namibia.

neighbour) as the main DOTS provider (39.4\%; table 2), were in the continuation phase of treatment (63.73\%) and were not taking ART (83.9\%). The cases of LTFU were significantly higher in regions with border, transit, and central business districts such as Khomas and Kunene ( $\mathrm{p}=0.001$, figures 3 and 4).

The univariate logistic regression analysis showed a significant association between percentage of cases of LTFU and young/middle-aged adult patients ( $15-45$ years $)(p=0.001)$, male sex $(p=0.001)$ and region where TB care was received ( $p=0.001)$, known HIV status $(p=0.001)$ and 2-month sputum conversion $(p=0.001)$. The medicine-related characteristics associated with LTFU: ART $(p=0.001)$, a relative being a

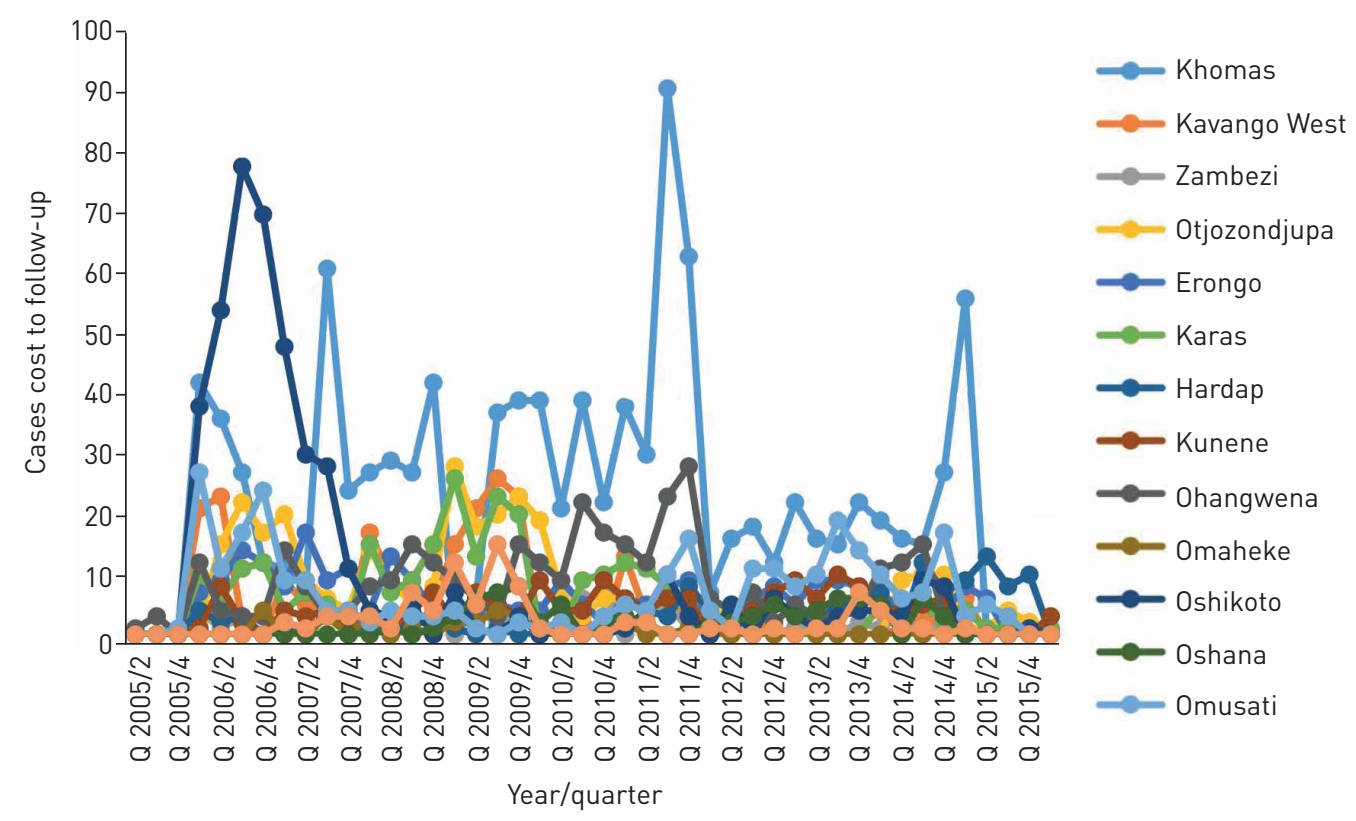

FIGURE 4 Absolute tuberculosis cases lost to follow-up by region in Namibia. 
TABLE 2 Characteristics and factors associated with tuberculosis (TB) cases lost to follow-up (LTFU) in Namibia

\begin{tabular}{|c|c|c|c|c|c|c|}
\hline \multirow[t]{2}{*}{ Characteristic } & \multirow[t]{2}{*}{ Total } & \multicolumn{2}{|c|}{ TB treatment outcome } & \multirow[t]{2}{*}{ df } & \multirow[t]{2}{*}{$\operatorname{cOR}(95 \% \mathrm{CI})$} & \multirow[t]{2}{*}{$\mathrm{p}$-value } \\
\hline & & Successful & LTFU & & & \\
\hline All TB cases & $65113(100.0 \%)$ & $61338(94.2 \%)$ & $3775(5.8 \%)$ & & & \\
\hline \multicolumn{7}{|l|}{ TB strateqy employed } \\
\hline MTP-I & $30771(100.0 \%)$ & $28766(93.5 \%)$ & $2005(6.5 \%)$ & \multirow[t]{2}{*}{1} & $1.3(1.2-1.4)$ & \multirow[t]{2}{*}{$0.000 *$} \\
\hline MTP-II & $34342(100.0 \%)$ & $32572(94.8 \%)$ & $1770(5.2 \%)$ & & 1 & \\
\hline \multicolumn{7}{|l|}{ Region } \\
\hline Khomas & $13137(100.0 \%)$ & $12105(92.1 \%)$ & $1032(7.9 \%)$ & \multirow[t]{14}{*}{13} & $1.1(0.9-1.4)$ & $0.000 *$ \\
\hline Kavango West & $4572(100.0 \%)$ & 4335 (94.8\%) & $237(5.2 \%)$ & & $0.7(0.5-0.9)$ & 0.410 \\
\hline Zambezi & $2788(100.0 \%)$ & $2726(97.8 \%)$ & $62(2.2 \%)$ & & $0.3(0.2-0.4)$ & $0.007^{*}$ \\
\hline Otjozondjupa & $4021(100.0 \%)$ & $3698(92.0 \%)$ & $323(8.0 \%)$ & & $1.1(0.9-1.4)$ & $0.000 *$ \\
\hline Erongo & $7852(100.0 \%)$ & $7588(96.6 \%)$ & $264(3.4 \%)$ & & $0.4(0.3-0.6)$ & 0.340 \\
\hline Karas & $4122(100.0 \%)$ & $3859(93.6 \%)$ & $263(6.4 \%)$ & & $0.9(0.7-1.1)$ & $0.000^{*}$ \\
\hline Hardap & $2660(100.0 \%)$ & $2522(94.8 \%)$ & $138(5.2 \%)$ & & $0.7(0.5-0.9)$ & 0.313 \\
\hline Kunene & $2069(100.0 \%)$ & $1897(91.7 \%)$ & $172(8.3 \%)$ & & $1.2(0.9-1.5)$ & $0.013^{*}$ \\
\hline Ohangwena & $6248(100.0 \%)$ & 5903 (94.5\%) & $345(5.5 \%)$ & & $0.8(0.6-1.0)$ & 0.251 \\
\hline Omaheke & $2847(100.0 \%)$ & $2822(99.1 \%)$ & $25(0.9 \%)$ & & $0.1(0.1-0.2)$ & $0.022 *$ \\
\hline Oshikoto & $6331(100.0 \%)$ & $5885(93.0 \%)$ & $446(7.0 \%)$ & & $0.9(0.8-1.2)$ & $0.000^{*}$ \\
\hline Oshana & 1593 (100.0\%) & $1508(94.7 \%)$ & $85(5.3 \%)$ & & $0.7(0.5-1.0)$ & 0.851 \\
\hline Omusati & $5650(100.0 \%)$ & $5355(94.8 \%)$ & $295(5.2 \%)$ & & $0.7(0.5-0.9)$ & $0.042^{*}$ \\
\hline Kavango East & $1223(100.0 \%)$ & $1135(92.8 \%)$ & $88(7.2 \%)$ & & 1 & $0.007^{*}$ \\
\hline \multicolumn{7}{|l|}{ Health facility } \\
\hline Hospital & $13334(100.0 \%)$ & $12348(92.6 \%)$ & $986(7.4 \%)$ & \multirow[t]{3}{*}{2} & $1.4(1.2-1.5)$ & $0.000 *$ \\
\hline PHC clinic & 41454 (100.0\%) & $39240(94.7 \%)$ & $2214(5.3 \%)$ & & $1.0(0.9-1-1)$ & $0.000 *$ \\
\hline Health centre & 10325 (100.0\%) & 9750 (94.4\%) & 575 (5.6\%) & & 1 & 0.358 \\
\hline \multicolumn{7}{|l|}{ Sex } \\
\hline Male & 37479 (100.0\%) & 34986 (93.3\%) & $2493(6.7 \%)$ & \multirow[t]{4}{*}{1} & $1.5(1.4-1.6)$ & \multirow[t]{2}{*}{$0.000^{*}$} \\
\hline Female & 27634 (100.0\%) & $26352(95.4 \%)$ & $1282(4.6 \%)$ & & 1 & \\
\hline Age years mean \pm sD & 1.27 & $33.5 \pm 16.9$ & $33.9 \pm 16.4$ & & & $0.000 *$ \\
\hline \multicolumn{6}{|l|}{ Age categories years } & \\
\hline $0-4$ & $4420(100.0 \%)$ & $4191(94.8 \%)$ & $229(5.2 \%)$ & \multirow[t]{8}{*}{7} & $0.9(0.7-1.1)$ & $0.000^{*}$ \\
\hline $5-14$ & 3901 (100.0\%) & $3729(95.6 \%)$ & 172 (4.4\%) & & $0.7(0.6-0.9)$ & 0.202 \\
\hline $15-24$ & $8251(100.0 \%)$ & 7766 (94.1\%) & 485 (5.9\%) & & $1.0(0.8-1.2)$ & $0.006^{*}$ \\
\hline $25-34$ & $18760(100.0 \%)$ & $17564(93.6 \%)$ & $1196(6.4 \%)$ & & $1.1(0.9-1.3)$ & 0.976 \\
\hline $35-44$ & $15447(100.0 \%)$ & $14567(94.3 \%)$ & $880(5.7 \%)$ & & $0.9(0.8-1.1)$ & 0.280 \\
\hline $45-54$ & $7734(0 \%)$ & $7292(94.3 \%)$ & $442(5.7 \%)$ & & $0.9(0.8-1.2)$ & 0.718 \\
\hline $55-64$ & $3547(100.0 \%)$ & $3355(94.6 \%)$ & $192(5.4 \%)$ & & $0.9(0.7-1.1)$ & 0.766 \\
\hline$\geqslant 65$ & $3053(100.0 \%)$ & $2874(94.1 \%)$ & $179(5.9 \%)$ & & 1 & 0.429 \\
\hline First-line TB regimen & & & & & & \\
\hline $2 \mathrm{HRZE} / 4 \mathrm{HRE}$ & $49226(100.0 \%)$ & $46673(94.8 \%)$ & $2553(5.2 \%)$ & & $0.7(0.4-1.1)$ & $0.000 *$ \\
\hline 2 HRZES/ 1 HRZE/5 HRE & $10846(100.0 \%)$ & $9883(91.1 \%)$ & $963(8.9 \%)$ & 4 & $1.2(0.8-2.0)$ & 0.135 \\
\hline 2 HRZS/1 HRZ/5 HR (children) & $304(100.0 \%)$ & $288(94.7 \%)$ & $16(5.3 \%)$ & & $0.7(0.4-1.4)$ & 0.360 \\
\hline 2 HRZ/4 HR (children) & $4475(100.0 \%)$ & $4251(95.0 \%)$ & $224(5.0 \%)$ & & $0.6(0.4-1.1)$ & 0.329 \\
\hline Other regimens & $262(100.0 \%)$ & $243(92.7 \%)$ & $19(7.3 \%)$ & & 1 & 0.111 \\
\hline TB case registered & & & & & & \\
\hline New & $53956(100.0 \%)$ & 51162 (94.8\%) & $2794(5.2 \%)$ & 5 & $0.7(0.6-0.8)$ & $0.000 *$ \\
\hline Failure & $402(100.0 \%)$ & $370(92.0 \%)$ & $32(8.0 \%)$ & & $1.2(0.8-1.7)$ & $0.000^{*}$ \\
\hline Other previously treated & $2769(100.0 \%)$ & $2508(90.6 \%)$ & $261(9.4 \%)$ & & $1.4(1.2-1.6)$ & 0.465 \\
\hline Readmission & $842(100.0 \%)$ & $674(80.0 \%)$ & $168(20.0 \%)$ & & $3.3(2.7-4.0)$ & $0.000^{*}$ \\
\hline Recurrent TB & 1069 (100.0\%) & $974(91.1 \%)$ & $95(8.9 \%)$ & & $1.3(1.1-1.6)$ & $0.000 *$ \\
\hline Relapse & $6075(100.0 \%)$ & $5650(93.0 \%)$ & $425(7.0 \%)$ & & 1 & 0.029 \\
\hline TB case & & & & & & \\
\hline New TB case & $53956(100.0 \%)$ & 51162 (94.8\%) & $2794(5.2 \%)$ & 1 & $0.6(0.5-0.6)$ & $0.000 *$ \\
\hline Previously treated & $11157(100.0 \%)$ & $10176(91.2 \%)$ & $981(8.8 \%)$ & & 1 & \\
\hline Pulmonary TB case & $52454(100.0 \%)$ & $49345(94.1 \%)$ & $3109(5.9 \%)$ & 1 & $1.1(1.0-1.2)$ & $0.004^{*}$ \\
\hline Extrapulmonary TB & $12659(100.0 \%)$ & 11993 (94.7\%) & $666(5.3 \%)$ & & 1 & \\
\hline
\end{tabular}




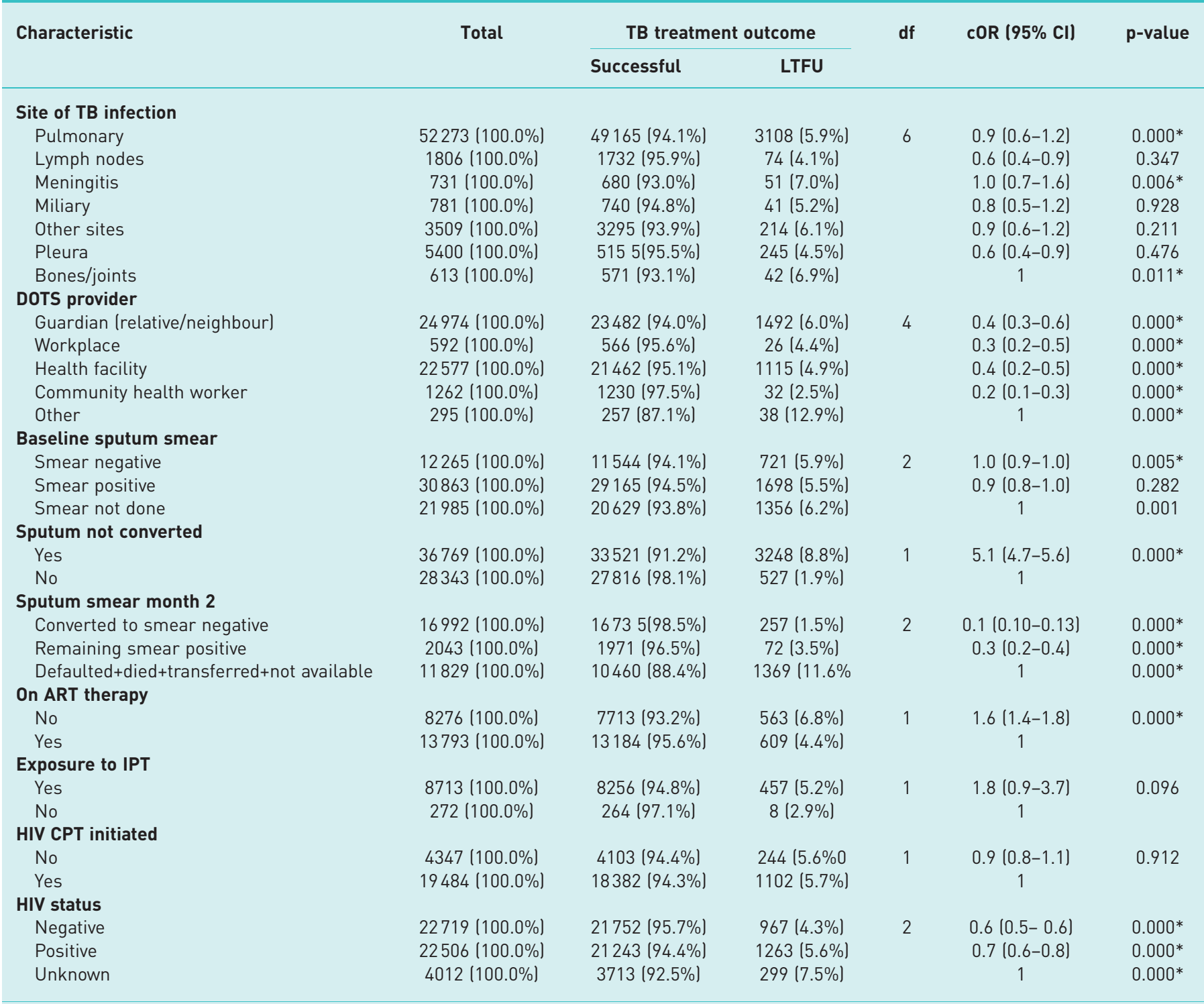

df: degrees of freedom; cOR: crude odds ratio; MTP: medium-term plan; PHC: primary healthcare; HRZE: isoniazid/rifampicin/pyrazinamide/ ethambutol; HRE: isoniazid/rifampicin/ethambutol; DOTS: directly observed treatment short-course; ART: anti-retroviral treatment; IPT: isoniazid preventive therapy; CPT: co-trimoxazole preventive therapy. * $\mathrm{p}<0.05$.

DOTS provider $(\mathrm{p}=0.001)$, type of TB regimen type $(\mathrm{p}=0.044)$, MoHSS MTP-I strategic plan $(\mathrm{p}=0.001)$, and level of healthcare facility for TB care $(\mathrm{p}=0.002)$ (table 2$)$.

Independent predictors of LTFU of the DOTS programme in Namibia

A multivariate logistic regression analysis was conducted to identify predictors of LTFU (table 3). A test of the full model against was statistically significant, indicating that the predictors as a set reliably distinguished between LTFU and non-LTFU outcomes $\left(\chi^{2}=36.6, p=0.001\right.$ with $\left.\mathrm{df}=15\right)$. Nagelkerke's $\mathrm{R}^{2}$ of 0.35 indicated a relationship between prediction and grouping by LTFU. Prediction success overall was $71.7 \%$ (60.8\% for LTFU and $79.7 \%$ for non-LTFU). The Wald criterion demonstrated that MTP-I strategy implemented by the National TB and Leprosy Program, male sex [19], type of DOTS provider [20], a 2-month sputum conversion; the region in Namibia of DOTS implementation; particularly Otjozondjupa, Karas and Kunene regions; and the young/middle-age categories (i.e. 15-45 years of age) made a significant contribution to prediction of LTFU. However, there was no further significant association of LTFU with HIV status, regimen type and level of DOTS facility. 


\begin{tabular}{|c|c|c|}
\hline Covariates & aOR $(95 \% \mathrm{CI})$ & p-value \\
\hline \multicolumn{3}{|l|}{ MTP for TB } \\
\hline MTP period I & $0.5(0.30-0.96)$ & $0.037^{*}$ \\
\hline MTP period II & 1 & \\
\hline \multicolumn{3}{|l|}{ Region } \\
\hline Karas & $18.8(4.9-73)$ & $0.001 *$ \\
\hline Otjozondjupa & $3.9(1.0-15.6)$ & $0.001^{*}$ \\
\hline Kunene & $3.2(1.0-4.1)$ & $0.048^{*}$ \\
\hline Khomas & $1.7(0.13-23)$ & $0.043^{*}$ \\
\hline Kavango West & $1.4(0.5-4.0)$ & 0.681 \\
\hline Zambezi & $1.4(0.5-4.0)$ & 0.491 \\
\hline Erongo & $0.4(0.04-3.8)$ & 0.443 \\
\hline Hardap & $0(0-10.4)$ & 0.430 \\
\hline Ohangwena & $0.6(0.1-7.3)$ & 1.000 \\
\hline Omaheke & $1.6(0.4-2.1)$ & 0.641 \\
\hline Oshikoto & $0(0)$ & 0.499 \\
\hline Oshana & $0.7(0.7)$ & 0.999 \\
\hline Kavango East & 1 & 0.537 \\
\hline \multicolumn{3}{|l|}{ Patient sex } \\
\hline Male & $2.2(1.3-3.8)$ & $0.004^{*}$ \\
\hline Female & 1 & \\
\hline \multicolumn{3}{|l|}{ Patient age years } \\
\hline $0-4$ & $0(0-451)$ & $0.025^{*}$ \\
\hline $5-14$ & $14.4(0.5-281)$ & 1.000 \\
\hline $15-24$ & $29.7(3.1-194)$ & 0.128 \\
\hline $25-34$ & $21.6(2.4-110)$ & $0.003^{*}$ \\
\hline $35-44$ & $12.2(1.4-109)$ & $0.006^{*}$ \\
\hline $45-54$ & $11.5(1.2-71.6)$ & $0.025^{*}$ \\
\hline $55-64$ & $6.2(0.6-0.8)$ & $0.033^{*}$ \\
\hline$\geqslant 65$ & 1 & 0.140 \\
\hline \multicolumn{3}{|l|}{ DOTS regimen } \\
\hline $2 \mathrm{RHZE} / 4 \mathrm{RHE}$ & $1.0(0.6-1.9)$ & 0.748 \\
\hline Other regimens & 1 & \\
\hline \multicolumn{3}{|l|}{ DOTS provider } \\
\hline Guardian & $1.0(0.3-4.0)$ & $0.001 *$ \\
\hline Workplace & $0.8(0.03-26.8)$ & 0.886 \\
\hline Health facility & $0.3(0.08-1.2)$ & 0.921 \\
\hline Community health worker & $0(0)$ & 0.085 \\
\hline Other DOTS providers & 1 & 0.999 \\
\hline \multicolumn{3}{|l|}{2 month's sputum conversion } \\
\hline Smear negative & $1.6(0.9-3.2)$ & $0.047^{*}$ \\
\hline Smear positive & $0.4(0.2-1.2)$ & 0.113 \\
\hline Smear not assessed & 1 & 0.116 \\
\hline \multicolumn{3}{|l|}{ Anti-retroviral treatment } \\
\hline No & $1.9(0.9-3.9)$ & 0.052 \\
\hline Yes & 1 & \\
\hline \multicolumn{3}{|l|}{ HIV status } \\
\hline Negative & $0.8(0.2-3.0)$ & 0.474 \\
\hline Positive & $1.5(0.4-6.2)$ & 0.776 \\
\hline Unknown & 1 & 0.563 \\
\hline \multicolumn{3}{|l|}{ Facility level } \\
\hline Hospital & $0.6(0.2-1.9)$ & 0.511 \\
\hline PHC clinic & $0.6(0.3-1.4)$ & 0.437 \\
\hline Health centre & 1 & 0.247 \\
\hline Constant & 0 & 0.006 \\
\hline \multicolumn{3}{|c|}{$\begin{array}{l}\text { aOR: adjusted odds ratio; MTP: medium-term plan; TB: tuberculosis; DOTS: directly observed treatment, } \\
\text { short-course; RHZE: rifampicin/isoniazid/pyrazinamide/ethambutol; RHE: rifampicin/isoniazid/ethambutol; } \\
\text { PHC: primary healthcare. *: } p<0.05 \text {. }\end{array}$} \\
\hline
\end{tabular}




\section{Discussion}

In the sample taken in this study, of all patients notified in Namibia that had a treatment outcome registered between 2006 and 2016, 1 out of every 25 TB cases registered was LTFU (table 2 and figure 2). Furthermore, one out of four patients with unsuccessful outcomes was a case of LTFU. The prevalence of LTFU varied widely between geographical regions in Namibia, $6.6 \%$ to $34 \%$. These are higher than the national (2\%) and global benchmarks for LTFU (0\%). Studies in other LMICs in Africa and Asia report the prevalence of LTFU to range between $6 \%$ and $24 \%[15,21]$. This calls for national and regional specific strategies to be incorporated in the medium and long-term strategic plans for TB to reduce the burden of LTFU. The strategy should target building capacity in tracing and supporting patients at risk of LTFU, particularly those registered for DOTS services in border/transit points as well as regions with central business districts.

Multivariate logistic regression suggested that the TB patients most at risk of LTFU were male patients, young adults $(<45$ years), cases registered in the capital city, patients in transit and/or close to geographical border DOTS access points with high TB notification rates. Several studies have linked LTFU to patients initiated on therapy at immigration towns like borders and transit points. Similarly to our findings, studies associate higher LTFU rates among male patients [22-24], the youth or middle aged (34-44 years) [16, 23]. Contrary to our findings, Peltzer et al. [25] found no association between LTFU and male sex in a population in South Africa. Nonetheless, young adults in LMICs are a high-risk group for unemployment, HIV, alcohol and drug abuse, smoking, multiple sexual partners that predispose then to TB and poor outcomes, such as LTFU [12-15]. Secondly, the study demonstrated that the clinical risk factors for LTFU were new TB cases, a diagnosis of pulmonary TB versus extrapulmonary $\mathrm{TB}$, and sputum conversion at 2 months (i.e. end of the intensive phase of treatment), but not HIV co-infection. Other studies found association of HIV [14] and regimen-related adverse drug reaction (ADR) [20] with LTFU among TB cases. Thirdly, the programmatic predictors of LTFU were interventions under the respective MTP strategy and DOTS support; MTP-II reduced LTFU by 50\% $(\mathrm{p}=0.037)$. There was no association between the treatment regimen, ART and previous IPT exposure, HIV status and CPT prophylaxis and the occurrence of LTFU ( $>0.05)$ (table 2).

Potential limitations of this study should be considered while interpreting the findings. Firstly, the retrospective data had missing information on the treatment outcome for several patients and were excluded from the analysis. In addition, we excluded cases with other poor treatment outcomes (i.e. death, transferred out and failure) and comparisons were made with patients who had a successful treatment outcome (i.e. cured and completed treatment). There may be many other factors which better explain LTFU that were not reported in the ETR database used. Nevertheless, the study utilises a nationwide dataset in a high-TB-burden country over a significant time period (10-year period). This nationwide study highlights the significance of improving socioeconomic welfare of TB patients to abate LTFU, as TB is a disease highly prevalent among patients of low socioeconomic backgrounds. Currently strategic goals of the TB programme in Namibia are mainly case identification and management with limited socioeconomic interventions among young adults in Namibia.

In conclusion, the study demonstrates a high prevalence LTFU among TB cases registered under the DOTS programme in Namibia, this is above the global target of $0 \%$. The findings imply that main factors driving LTFU are related to socioeconomic welfare of young adults who seek temporary employment in regions that provide temporary working opportunities, including borders and capital towns. This age group is an important driver for HIV infection in Namibia. The study recommends the integration of socioeconomic interventions/incentives in DOTS programmes to support young adult TB cases in informal and/or temporary employments across all regions in Namibia. In addition, there is a need for integration of DOTS services in workplaces and institutions that provide temporary employment (e.g. construction sites) for mobile young male adults to enhance continuity of TB treatment and improve outcomes.

Conflict of interest: None declared.

\section{References}

1 Lawn L, Myer L, Bekker LG, et al. Burden of tuberculosis in an antiretroviral treatment programme in sub-Saharan Africa: impact on treatment outcomes and implications for tuberculosis control. AIDS 2006; 20: $1605-1612$

2 Jones CL. HHS public access. Department of Health and Human Services Unites States of America 2015; 33: 395-401.

3 World Health Organisation. Global Tuberculosis Report. Geneva: World Health Organisation, 2019. Licence: CCBY-NC-SA3.0IGO

4 Woldeyes BG. Evaluation of directly observed tuberculosis treatment strategy in Ethiopia: patient centeredness and satisfaction. [Thesis]. University of South Africa, 2016. http://hdl.handle.net/10500/22074. 
Republic of Namibia Ministry of Health and Social Services. Republic of Namibia Ministry of Health and Social Services National Tuberculosis and Leprosy Programme Annual Report: 2014-2015. Windhoek Namibia, MoHSS, 2015.

$6 \quad$ World Health Organization. Global Tuberculosis Report 2016. Geneva, WHO, 2016.

7 van Gorkom J, Mavhunga F, Omer OA, et al. TB control in Namibia 2002-2011: progress and technical assistance. Open Infect Dis J 2013; 7: 23-29.

8 Republic of Namibia Ministry of Health and Social Services. National Strategic Plan on Tuberculosis (TB) Medium-Term Plan I (MTP-I): 2004-2009. Windhoek, MoHSS, 2004.

9 Republic of Namibia Ministry of Health and Social Services. National Strategic Plan on Tuberculosis (TB), Medium-Term Plan II (MTP-II): 2010-2015. Windhoek, MoHSS, 2010.

10 Republic of Namibia Ministry of Health and Social Services. National Tuberculosis and Leprosy Programme Summary Report 2014-15. Windhoek, Namibia, MoHSS, 2015.

11 Li MS, Musonda P, Gartland M, et al. Predictors of patient attrition according to different definitions for loss to follow-up: a comparative analysis from Lusaka, Zambia. J Acquir Immune Defic Syndr 2013; 63: e116-e119.

12 World Health Organization. Global Tuberculosis Report 2014. Geneva, World Health Organization, 2014.

13 MacPherson P, Houben RM, Glynn JR, et al. Pre-treatment loss to follow-up in tuberculosis patients in low- and lower-middle-income countries and high-burden countries: a systematic review and meta-analysis. Bull World Health Organ 2014; 92: 126-138.

14 Akessa GM, Tadesse M, Abebe G. Survival analysis of loss to follow-up treatment among tuberculosis patients at Jimma University Specialized Hospital, Jimma. Southwest Ethiopia Int J Stat Mech 2015; 2015: 1-7.

15 Heemanshu A, Satwanti K. Determinants of lost to follow up during treatment among tuberculosis patients in Delhi. Int J Med Res Health Sci 2016; 5: 145-152.

16 Liew SM, Khoo EM, Ho BK, et al. Tuberculosis in Malaysia: predictors of treatment outcomes in a national registry. Int J Tuberc Lung Dis 2015; 19: 764-771.

17 World Health Organization. Global Tuberculosis Report 2016. Geneva: World Health Organization, 2016.

18 Strom BL, Kimmel SE, Hennessy S. Pharmacoepidemiology, 5th Edn. John Wiley \& Sons, 2013.

19 Pepper D, Schomaker M, Wilkinson R, et al. Predictors of loss to follow-up during TB treatment in a high HIV prevalence setting. Am J Respir Crit Care Med 2013; 187: A1673.

20 Tupasi TE, Garfin AMCG, Kurbatova E V, et al. Factors associated with loss to follow-up during treatment for multidrug-resistant tuberculosis, the Philippines, 2012-2014. Emerging Infect Dis 2016; 22: 491-502.

21 Tran DA, Ngo AD, Shakeshaft A, et al. Trends in and determinants of loss to follow up and early mortality in a rapid expansion of the antiretroviral treatment program in Vietnam: findings from 13 outpatient clinics. PLoS ONE 2013; 8: e73181.

22 Wohlleben J, Makhmudova M, Saidova F, et al. Risk factors associated with loss to follow-up from tuberculosis treatment in Tajikistan: a case-control study. BMC Infect Dis 2017; 17: 543.

23 Shringarpure KS, Isaakidis P, Sagili KD, et al. Loss-to-follow-up on multidrug resistant tuberculosis treatment in Gujarat, India: the when and who of it. PLoS ONE 2015; 10: e0132543.

24 Namuwenge PM, Mukonzo JK, Kiwanuka N, et al. Loss to follow up from isoniazid preventive therapy among adults attending HIV voluntary counseling and testing sites in Uganda. Trans R Soc Trop Med Hyg 2012; 106: 84-89.

25 Peltzer K, Louw JS. Prevalence and associated factors of tuberculosis treatment outcome among hazardous or harmful alcohol users in public primary health care in South Africa. Afr Health Sci 2014; 14: 157-166. 